\title{
Quantitative Evaluation of Mixing Characteristics of Static Mixers by Visualization Experiments
}

\author{
Noboru Mochizuki' ${ }^{1}$, Aya Kaide ${ }^{2}$, Takashi Saeki2* \\ ${ }^{1}$ Business Development Department, ISEL Co., Ltd., Osaka, Japan \\ ${ }^{2}$ Graduate School of Science and Technology for Innovation, Yamaguchi University, Yamaguchi, Japan \\ Email: *saeki@yamaguchi-u.ac.jp
}

How to cite this paper: Mochizuki, N., Kaide, A. and Saeki, T. (2018) Quantitative Evaluation of Mixing Characteristics of Static Mixers by Visualization Experiments. Journal of Flow Control, Measurement \& Visualization, 6, 27-38.

https://doi.org/10.4236/jfcmv.2018.61003

Received: February 1, 2017

Accepted: December 31, 2017

Published: January 3, 2018

Copyright $\odot 2018$ by authors and Scientific Research Publishing Inc. This work is licensed under the Creative Commons Attribution International License (CC BY 4.0). http://creativecommons.org/licenses/by/4.0/

\begin{abstract}
We propose a convenient way of evaluating the mixing performance of static mixers used for round pipe by conducting flow visualization experiments under the turbulent region and using water as the main stream. A fluorescent pigment, glycerin, two carboxymethyl cellulose solutions, and rapeseed oil were each injected upstream of the mixer. Three static mixer conditions were tested: 1) no static mixer; 2) a Kenics-type static mixer; and 3) a multi-stacked elements (MSE) static mixer. The mixing trend downstream of the mixer in each condition and with each injection fluid was monitored using a laser and high-speed video camera system to obtain cross-sectional images. We propose suitable indexes based on the images obtained for quantitative evaluations of the mixing characteristics of static mixers.
\end{abstract}

\section{Keywords}

Line Mixer, Stacked Elements, Pigment, Mixing Performance, Visualization Experiment

\section{Introduction}

A static mixer or line mixer is a device designed to mix fluids inside a pipe. Static misers are implanted in pipes, and can divide, shear, compress, and/or replace fluids passing over a short distance; they are therefore considered space-saving equipment compared to a stirring vessel. Many types of static mixers are commercially available. Familiar static mixer providers include Kenics Co., Charles Ross \& Son Co., and the Mixing Equipment Co. in the U.S. and the Sulzer Co. in Switzerland [1] [2]. In Japan, Noritake Co., OHR Co., Fujikin Co., and Toray 
Co. offer original static mixers. Since static mixers include no driving mechanism, they might be energy-saving; however, insufficient mixing often occurs due to the lack of retention time. It is thus important to develop new static mixers that provide better mixing performance.

At the same time, it is necessary to develop a method for evaluating mixing characteristics quantitatively, as these data could be used both for developing new static mixers and for selecting suitable candidates. Etchells et al. [3] proposed using a coefficient of variation, $\mathrm{CoV}$, as an index of mixing performance, which is defined as the standard deviation of the concentration distribution in the mixing field. Since the concept of the standard deviation can be applied only for a normal distribution, the numerical value and mixing image obtained by a visualization experiment could be expected to show some gaps. Alberini et al. [4] evaluated the performance of Kenics KM static mixers using two shear-thinning fluid streams in a visualization experiment. They adopted planar laser-induced fluorescence (PLIF) to obtain striation-like images at the mixer outlet by doping one fluid stream with a fluorescent dye upstream of the mixer inlet, and they then extracted the concentration distribution of the fluorescent dye. In another study, Alberini et al. proposed the areal distribution method [5], which characterizes individual striations by determining the distribution as a function of size and concentration. They concluded that their method gave a more consistent measure of mixing performances than the CoV. However, their experiments were conducted under only a laminar flow regimen, which allowed a characteristic pattern of the fluorescent dye.

In the present study, we conducted flow visualization experiments under a turbulent region using water as the main stream. Glycerin, carboxymethyl cellulose solutions, and rapeseed oil with a fluorescent pigment were injected upstream of the mixer. We examined two static mixers, and proposed suitable indexes obtained from the cross-sectional images that enabled quantitative characterization of mixing performance.

\section{Experimental Procedure}

\subsection{Static Mixers}

We developed a multi-stacked elements (MSE) mixer [6] comprised of two types of mixing elements (A and $\mathrm{B}$ ) as shown in Figure 1. The mixing elements were stacked and sandwiched between the middle plate and two blind plates. Figure 2 contains an image of the streamlines inside the MSE mixer through a pipe. The fluid enters from the gap between the MSE and the wall inside the pipe, and flows through a complicated flow channel formed by the stacked configuration. The fluid then goes through the hole in the middle plate and again flows through complicated channels, consequently enhancing the mixing performance. An MSE mixer with 10 pairs of elements stacked at both ends of the middle plate was used as the test device. A Kenics-type static mixer (NS: 1-N60-331-N, Noritake Co.), as shown in Figure 3, was also examined in this study. This static 


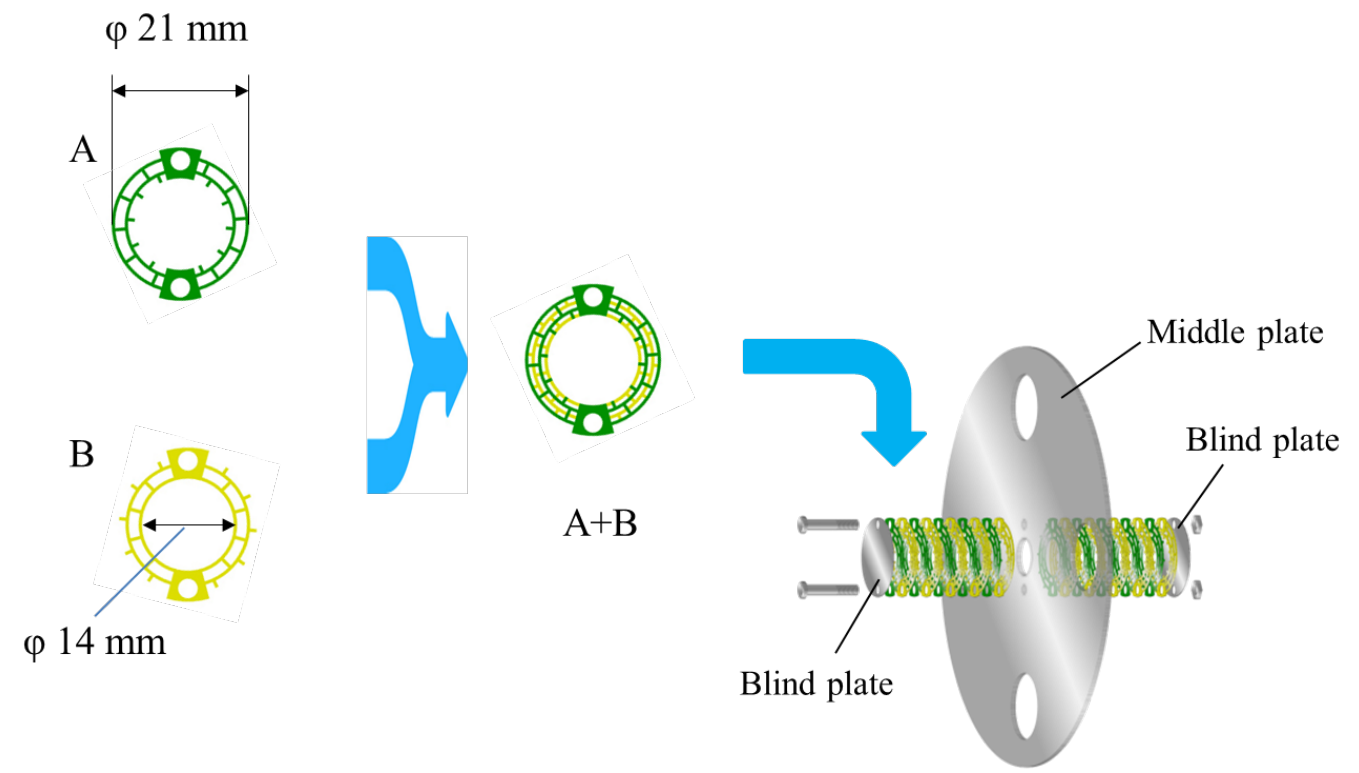

Figure 1. Assembly drawing for MSE.

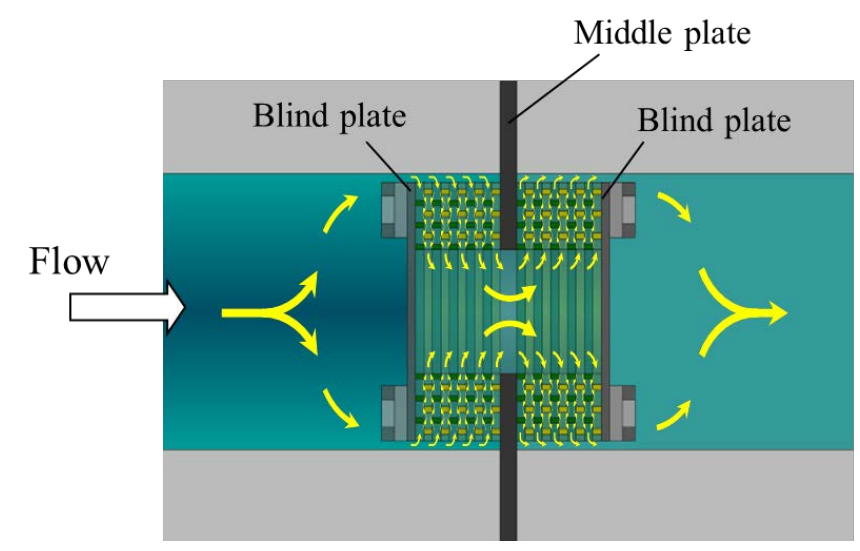

Figure 2. Image of streamlines inside the MSE mixer.

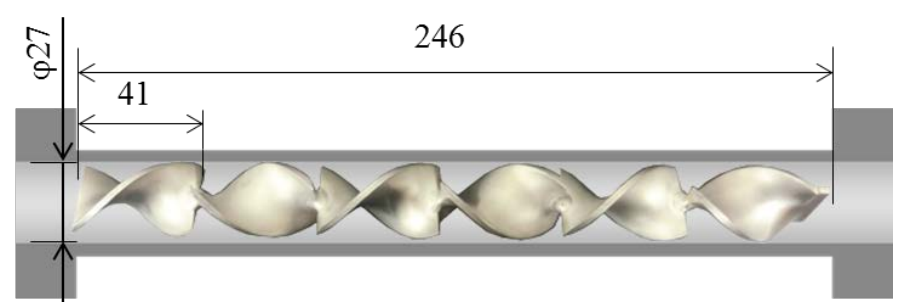

Figure 3. Schematic drawing of a Kenics-type static mixer.

mixer can be used for blending under both laminar and turbulent conditions by inducing circular patterns that reverse direction at each element's intersection.

\subsection{Flow System}

The experimental apparatus used in this study is shown in Figure 4. Tap water was continuously added to a tank and transported by a pump through a pipeline with a diameter of $25 \mathrm{~mm}$. The flow rate was measured with an electromagnetic 
flowmeter (FD-83, Keyence Co.), set at $0.5 \mathrm{~m} / \mathrm{s}$. The water temperature was $25^{\circ} \mathrm{C}$ for all of the experiments. The Reynolds number was calculated as 11,700 , using the diameter of the pipe as the reference length and the properties of water at $25^{\circ}$ C. Glycerin (Wako Co.) and carboxymethyl cellulose (CMC2260, Daicel Co.) solutions with different concentrations $(0.75,1.50 \mathrm{wt} \%)$, rapeseed oil (100\% lipid including 7\% saturated fatty acid, Nisshin Ollio Co.) were prepared as injected fluids. A fluorescent pigment, Neolan Pink B (Intracolor Co.) was added to injected fluids at the concentration of $1.0 \mathrm{wt} \%$.

The flow equilibrium curves of the injected fluids measured by a rheometer (NRM-2000R, Elquest Co.) are shown in Figure 5. Glycerin and the rapeseed oil were Newtonian fluids, whereas the CMC solutions showed non-Newtonian characteristics. They were injected by a micro-feeder through a nozzle (inner dia. $=3 \mathrm{~mm}$ ) at the center of the pipe, which was located $70-\mathrm{mm}$ upstream from the inlet of the static mixers. The viscosities of the CMC solutions at the shear

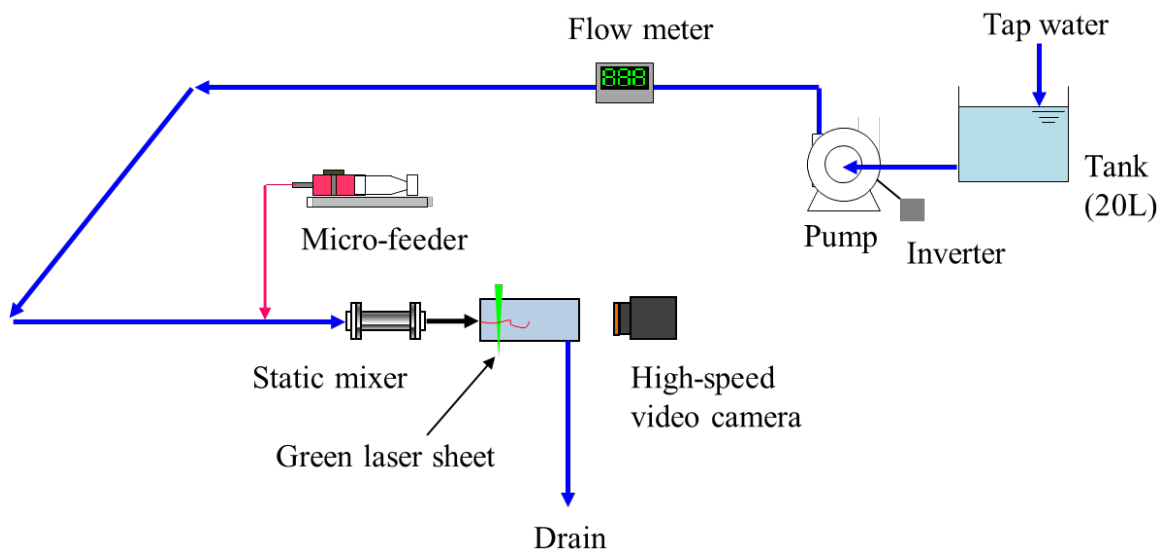

Figure 4. Flow systems.

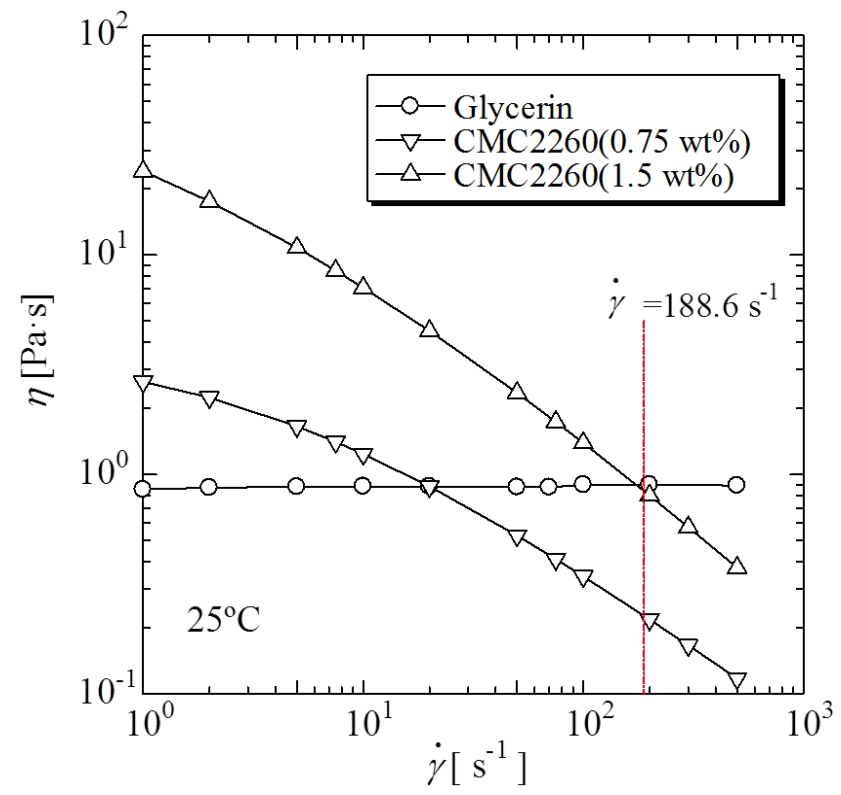

Figure 5. Flow equilibrium curves for injected fluids. 
rate equivalent to the outlet of the nozzle $\left(=188.6 \mathrm{~s}^{-1}\right)$ were $0.233 \mathrm{~Pa} \cdot \mathrm{s}$ for 0.75 wt $\%$ and $0.870 \mathrm{~Pa} \cdot \mathrm{s}$ for $1.50 \mathrm{wt} \%$, respectively. The flow rate of the injected fluid was set constant at $0.1 \mathrm{wt} \%\left(15 \mathrm{~cm}^{3} / \mathrm{min}\right)$ with respect to the main flow.

\subsection{Flow Visualization}

Figure 6 presents a schematic diagram and a photo of the flow visualization section set downstream of the static mixers. A transparent PVC pipe was connected just after the static mixers. The thickness of the PVC pipe was $0.2 \mathrm{~mm}$, to reduce the influence of the refraction of the round pipe wall. The cross-section $180-\mathrm{mm}$ downstream from the outlet of the static mixer was visualized by irradiating a $50-\mathrm{mW}$ green laser sheet with a wavelength of $532 \mathrm{~nm}$ at a right angle to the pipe. Distribution images of the injected fluid were captured by a high-speed video camera (VW-6000, Keyence Co.) at 1/60 frames for $15 \mathrm{~s}$ through a sharp-cut filter, the transmission limit wavelength of which was $560 \mathrm{~nm}$.

We converted the images $(330 \times 330$ pixels $)$ obtained into the quantitative values of RGB brightness (0 - 255) using ImageJ software (U.S. National Institutes of Health). We then determined the correlations between: 1) the concentration of fluorescent pigment and R brightness; 2) the concentration of fluorescent pigment and G brightness; and 3) the concentration of fluorescent pigment and $\mathrm{B}$ brightness. The results demonstrated that the concentration of fluorescent pigment and $\mathrm{R}$ brightness showed a good correlation [6]. Therefore, we used R-brightness for the mixing evaluation in this study. Although $G$ brightness also displayed good linearity, the presence of bubbles (which induced green emission) always caused non-negligible errors for $\mathrm{G}$ brightness values. We confirmed that more than 50 images were necessary to obtain stable statistical values (errors of less than 5\%). Therefore, we transacted 100 images for each experimental condition.

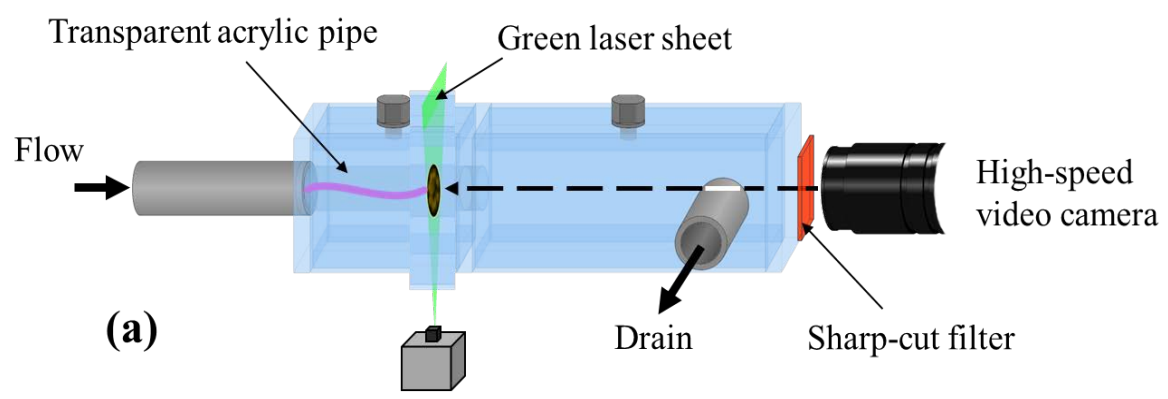

(b)

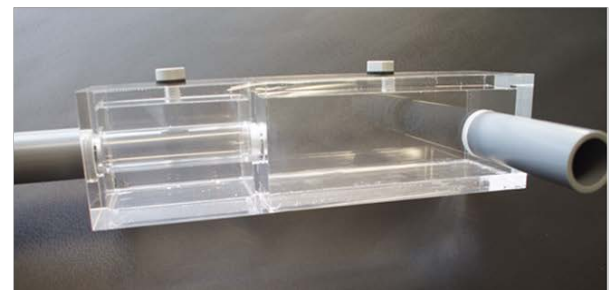

Figure 6. Flow visualization section: (a) schematic diagram: (b) photo. 


\subsection{Definition of Indexes}

We defined the following indexes to evaluate the mixing state of the obtained images:

1) Mixing rate: $M(\%)$

$M$ was defined as a spreading index, and was calculated by the number of fluorescent pixels divided by the total number of pixels inside the pipe cross-section (85,636 pixels). To take into account the white noise presented at the lower brightness region, the criterion of 30 for $\mathrm{R}$ brightness was used to judge whether or not a pixel was fluorescing. The threshold was set by comparing each $\mathrm{R}$ value with the corresponding pixel, and determining whether it was brightening or disappearing by viewing.

2) Extracted maximum brightness: $R_{\max }^{\prime}(-)$

$R_{\max }^{\prime}$ was introduced to extract highly concentrated lumps of the injected fluid. $n(R)$ was defined as the number of pixels for which the $\mathrm{R}$ brightness was $R$. Then, $n^{\prime}(R)$ was calculated using the following equation:

$$
n^{\prime}(R)=n(R)^{R / 255}
$$

$R_{\max }^{\prime}$ was then determined as the R brightness at which $n^{\prime}(R)$ was the maximum. Figure 7 shows $n^{\prime}(R)$ with respect to $R$ when $n(255)=100$. The presence of 100 pixels at $R=255$ is equivalent to 354, 125,892, and 6,309,573 pixels at $R=200,100$, and 75 , respectively. Therefore, we could extract the presence of higher-brightness pixels.

3) Size of bright lump: $L$ (\%)

$L$ was introduced to probe the presence of concentrated lumps, and we calculated the rate of brighter lump pixels to total pixels. Five bright lumps in order of size were chose for each image, and $L$ values were calculated.

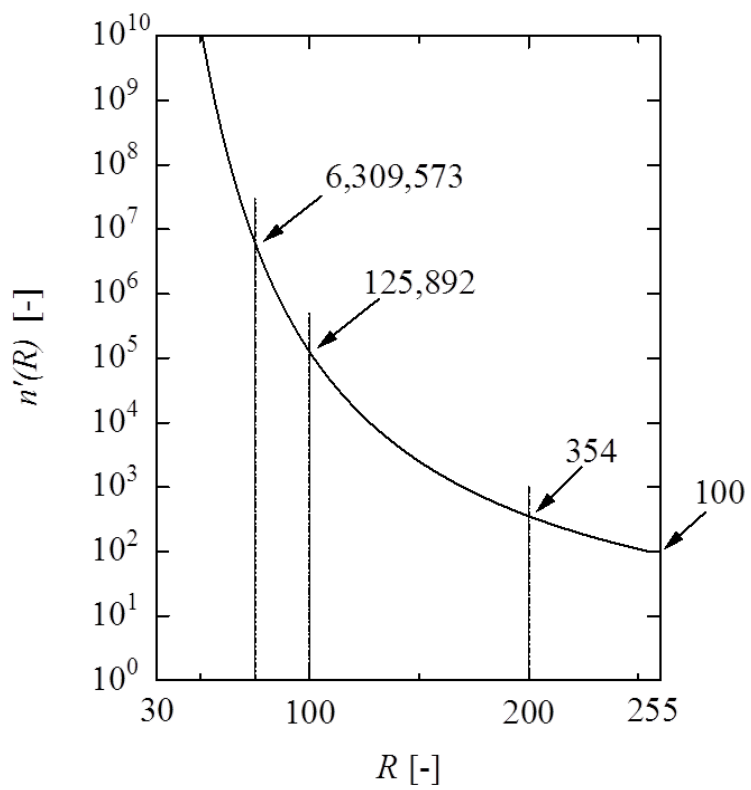

Figure 7. $n^{\prime}(R)$ vs. $R$. in the case of $n(255)=100$. 


\section{4) Deviation index: $\operatorname{Dev}(-)$}

We obtained the center of brightness using the ImageJ image-processing program, and the value of the y-coordinate was converted to Dev. Dev was defined to express the asymmetrical presence of the vertical direction of fluorescent pixels, where the Dev values at the top, center, and bottom inside the pipe were defined as 1,0 , and -1 , respectively. When the density of an injected fluid was larger than water at a certain level, a negative value of Dev might be obtained.

\section{Results and Discussion}

Figure 8 shows typical cross-sectional images for the static mixer conditions no mixer ("none"), the Kenics type static mixer (NS), and the MSE static mixer (MSE), obtained using glycerin as an injected fluid. We can see a large fluorescent lump located lower than the center for "none", while mixing was performed uniformly by the NS and MES mixers.

Table 1 shows the averaged index values calculated from 100 images for each static mixer condition. The $M$-values $>90 \%$ demonstrate the impressive mixing performance of the two static mixers. The "none" condition also showed a high $M$-value due to the high solubility of glycerin in water; however, the maximum value of $R_{\max }^{\prime}$ indicates the presence of an undiluted solution of glycerin. Similar values of $D e v$ among the three suggest that the density difference between glycerin and water did not affect mixing performance. With regard to $L$-values for "none" with glycerin, the largest lump occupied $13.39 \%$ of the total cross-section of the pipe. The second largest lump was only $0.06 \%$, and no other lumps were confirmed. We did not observe any lumps when the NS or MSE mixers were used.

Figure 9 shows typical images for the "none", NS, and MSE experiments obtained with the $0.75 \mathrm{wt} \%$ and $1.50 \mathrm{wt} \% \mathrm{CMC}$ solutions. Since the density of 0.75 $\mathrm{wt} \% \mathrm{CMC}$ is almost the same as that of water, the large lump remained almost at

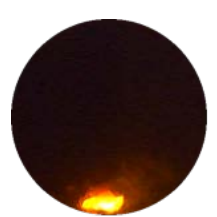

'none'

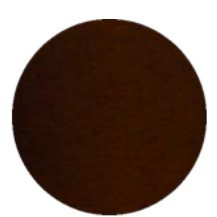

NS

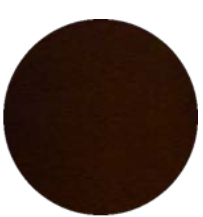

MSE

Figure 8. Cross-sectional images of "none", NS, and MSE for glycerin as an injected fluid.

Table 1. Mixing characteristics for glycerin evaluated by three indexes.

\begin{tabular}{cccc}
\hline \multirow{2}{*}{ Index } & \multicolumn{3}{c}{ Static mixer } \\
\cline { 2 - 4 } & "none" & NS & MSE \\
\hline$M(\%)$ & 64.58 & 97.38 & 96.30 \\
$R_{\max }^{\prime}(-)$ & 164 & 47 & 43 \\
$D e V(-)$ & -0.11 & -0.01 & -0.01 \\
\hline
\end{tabular}




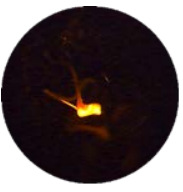

'none'

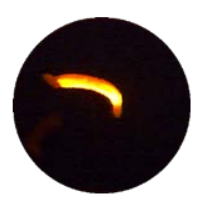

'none'

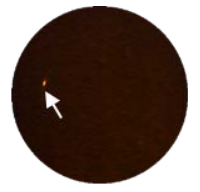

NS

(a)

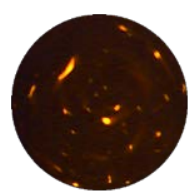

NS

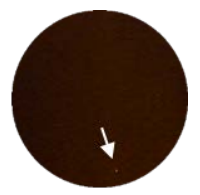

MSE

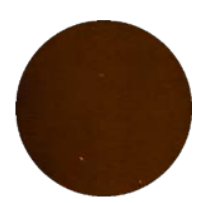

MSE

(b)

Figure 9. Cross-sectional images of "none", NS, and MSE with CMC solutions as injected fluids: (a) 0.75 wt $\%$ CMC: (b) $1.50 \mathrm{wt} \%$ CMC.

Table 2. Mixing characteristics for CMC solutions evaluated by three indexes.

0.75 wt $\%$ CMC

\begin{tabular}{|c|c|c|c|}
\hline \multirow{2}{*}{ Index } & \multicolumn{3}{|c|}{ Static mixer } \\
\hline & “none" & NS & MSE \\
\hline$M(\%)$ & 20.11 & 76.86 & 96.30 \\
\hline$R_{\max }^{\prime}(-)$ & 210 & 45 & 51 \\
\hline $\operatorname{Dev}(-)$ & 0.04 & -0.01 & 0.00 \\
\hline $\operatorname{CoV}(-)$ & 0.66 & 0.09 & 0.10 \\
\hline \multicolumn{4}{|c|}{$1.50 \mathrm{wt} \% \mathrm{CMC}$} \\
\hline \multirow{2}{*}{ Index } & \multicolumn{3}{|c|}{ Static mixer } \\
\hline & "none" & NS & MSE \\
\hline$M(\%)$ & 3.43 & 51.17 & 92.55 \\
\hline$R_{\max }^{\prime}(-)$ & 169 & 238 & 59 \\
\hline $\operatorname{Dev}(-)$ & -0.04 & -0.01 & -0.01 \\
\hline $\operatorname{CoV}(-)$ & 0.60 & 0.65 & 0.12 \\
\hline
\end{tabular}

the center of the pipe without much spreading for "none". In the NS and MSE cases, we observed that almost perfect mixing was achieved except for a few small pieces. In contrast, for the experiment using $1.50 \mathrm{wt} \% \mathrm{CMC}$ solution, the difference in mixing performance between the NS and MSE mixers was clear. The concentrated solution was subdivided by the NS mixer and remained as many pieces, whereas the MSE mixer dispersed the solution uniformly over the cross-section leaving only a few pieces.

Table 2 lists the averaged index values calculated from 100 images for each static mixer condition. Even the use of the MSE achieved higher $M$-values for $0.75 \mathrm{wt} \% \mathrm{CMC}$, the lower $R_{\max }^{\prime}$ values of NS and MSE indicated that the con- 
centrations of small pieces in both images were dilute. For $1.50 \mathrm{wt} \% \mathrm{CMC}$, the $M$-values indicate that the MSE showed better mixing characteristics than the NS, and our impression from these cross-sectional images supported this. The lowest value of $R_{\max }^{\prime}$ indicated high-performance mixing by the MSE. The reason for the zero Devvalue for "none" can be explained by the small difference in density between water and the CMC solutions. In contrast, the swirling flow that occurred when the NS mixer was used enhanced the mixing effect, consequently showing a zero $\mathrm{Dev}$ value. $\mathrm{CoV}$ values were also calculated for each CMC concentration and are given in the same table. Since larger values of $\mathrm{CoV}$ can be interpreted as lower mixing performance, the evaluation in the case of $0.75 \mathrm{wt} \%$ CMC might be reasonable. Meanwhile the mixing performance of "none" and NS were similar according to the $\mathrm{CoV}$ values for $1.50 \mathrm{wt} \% \mathrm{CMC}$, which showed some gaps with the image obtained by the visualization experiment. We considered that $\mathrm{CoV}$ deviated significantly when we observed the heterogeneous existence of thick threads and/or large lumps of the injected solution.

Figure 10 displays $L$-values in order of size for the "none", NS, and MSE mixers with the different CMC concentrations. These quantitations fit the impression of the mixing images obtained and shown in Figure 8.

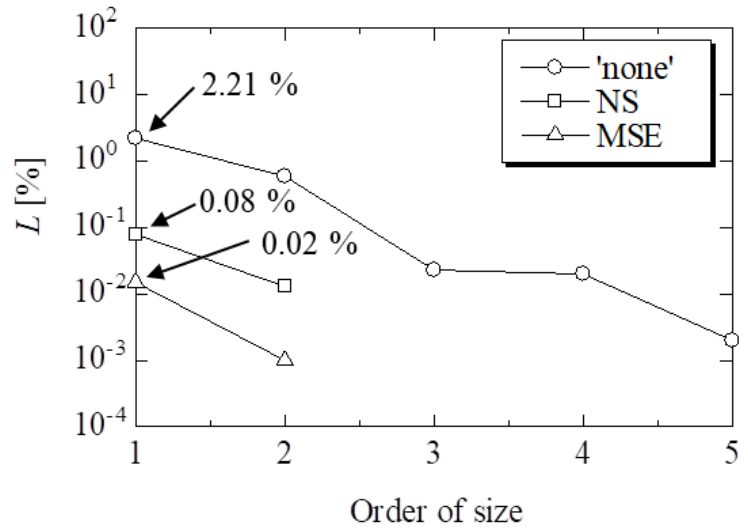

(a)

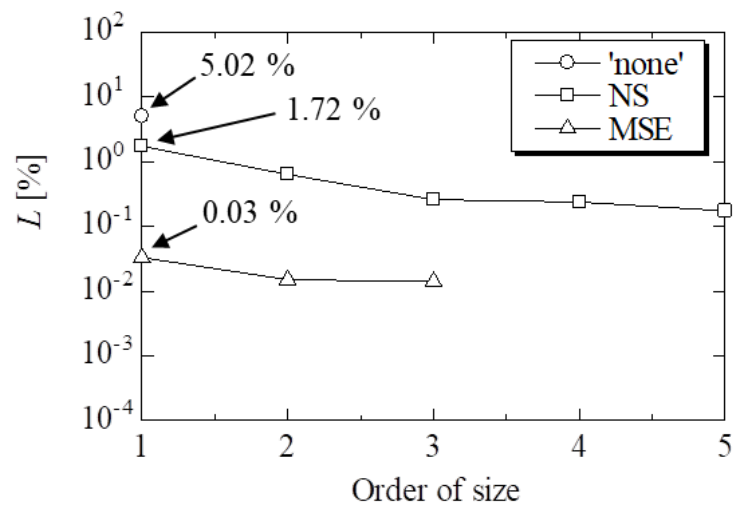

(b)

Figure 10. $L$ values in order of size for each static mixing conditions: (a) $0.75 \mathrm{wt} \% \mathrm{CMC}$; (b) $1.50 \mathrm{wt} \% \mathrm{CMC}$. 
Figure 11 shows typical cross-sectional images obtained for the rapeseed oil with the fluorescent pigment as an injected fluid. The size of the droplets decreased in the order "none", NS, and MSE; however, the differences in the brightness of droplets among the three mixer conditions were not significant. Since rapeseed oil does not dissolve in water, fluorescent oil droplets can be observed in the visualization experiment, and the concentration does not reflect the brightness. The particle-size distributions of the droplets can therefore be used as an alternative to evaluate the mixing performance for water-rapeseed oil systems.

Figure 12 displays the particle-size distributions of droplets obtained for the images shown in Figure 11. The 10\%, 50\%, and 90\% pass diameters of droplets for "none", NS, and MSE are also summarized. The lowest $D_{50}$ value and the smallest difference between $D_{10}$ and $D_{90}$ (the sharp distribution) demonstrate the high performance of the MSE. An MSE can be used as an emulsification apparatus by increasing the flow rate of the main flow of water. Under these conditions however, the mixing fluids become clouded, which hampers the visualization experiment proposed in this study.

In this paper, we considered the mixing characteristics of static mixers by observing injected CMC solutions with pigment. Although our proposed method is still a relative evaluation among various static mixers, it is an attempt to evaluate mixing characteristics quantitatively. The ability of a static mixer should be judged according to its mixing characteristics, flow resistance, cost, length, and so on.

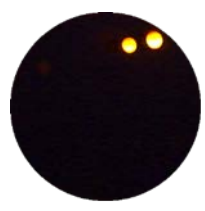

'none'

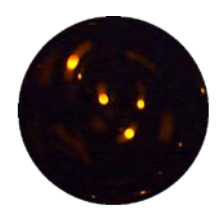

NS

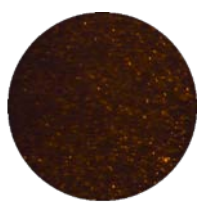

MSE

Figure 11. Cross-sectional images for "none", NS, and MSE for rapeseed oil as the injected fluid.

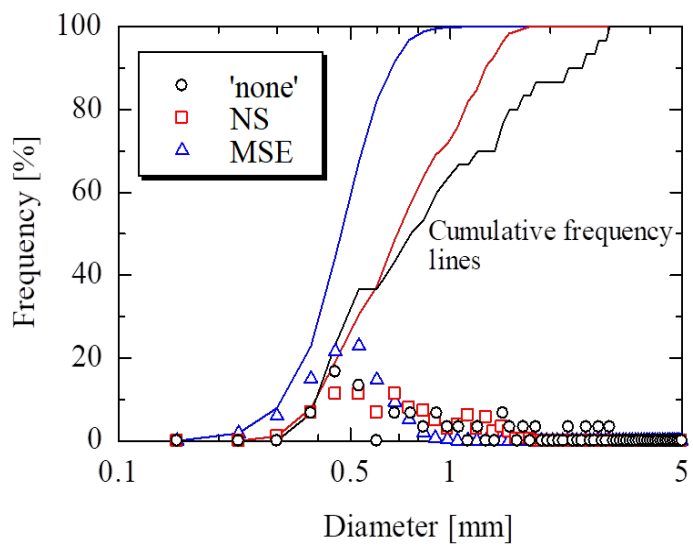

\begin{tabular}{cccc}
\hline & 'none' & NS & MSE \\
\hline$D_{10}(\mathrm{~mm})$ & 0.45 & 0.45 & 0.38 \\
$D_{50}(\mathrm{~mm})$ & 0.76 & 0.75 & 0.53 \\
$D_{90}(\mathrm{~mm})$ & 2.27 & 1.28 & 0.68 \\
\hline
\end{tabular}

Figure 12. Particle size distributions of oil droplets. 


\section{Conclusion}

We conducted flow visualization experiments for NS and MSE static mixers with the goal of enabling quantitative characterization of mixing performance. The indexes of $M, R_{\max }^{\prime}, L$, and Dev were defined in this study. The quantitative values of these indexes almost perfectly coincided with the impression of images obtained of the static mixers. We found that particle-size distribution is convenient for evaluations of mixing performance when a non-aqueous liquid is used as an injected fluid. Our analysis of the cross-sectional images obtained from the visualization experiments showed that the MSE provided effective mixing.

\section{Acknowledgements}

The authors appreciate the experimental assistance of a student colleague (Y. Moriguchi).

\section{References}

[1] Thakur, R.K., Vial, Ch., Nigam, K.D.P., Nauman, E.B. and Djelveh, G. (2003) Static Mixers in the Process Industries-A Review. Chemical Engineering Research and Design, 81, 787-826. https://doi.org/10.1205/026387603322302968

[2] Ghanem, A. Lemenand, T., Valle, D.D. and Peerhossaini, H. (2014) Static Mixers: Mechanisms, Applications, and Characterization Methods: A Review. Chemical Engineering Research and Design, 92, 205-228. https://doi.org/10.1016/j.cherd.2013.07.013

[3] Etchells, A.W. and Meyer, C.F. (2004) Mixing in Pipelines. In: Paul, E.L., Atiemo-Obeng, V.A. and Kresta, S.M., Eds., Handbook of Industrial Mixing, Wiley-Interscience, Hoboken, NJ.

[4] Alberini, F., Simmons, M.J.H., Ingram, A. and Stitt, E.H. (2014) Assessment of Different Methods of Analysis to Characterise the Mixing of Shear-Thinning Fluids in a Kenics KM Static Mixer Using PLIF. Chemical Engineering Science, 112, 152-169. https://doi.org/10.1016/j.ces.2014.03.022

[5] Alberini, F., Simmons, H.J.H., Ingram, A. and Stitt, E.H. (2014) Use of an Areal Distribution of Mixing Intensity to Describe Blending of non-Newtonian Fluids in a Kenics KM Static Mixer Using PLIF. AIChE Journal, 60, 332-342. https://doi.org/10.1002/aic.14237

[6] Mochizuki, N., Kurusu, T., Moriguchi, Y., Kaide, A. and Saeki, T. (2017) Development and Evaluation of Mixing Characteristic of a Static Mixer with Multi-Stacked Elements (MSE). Kagakukougaku Ronbunshu, 43, 75-80. (in Japanese) 


\section{Nomenclature}

$C_{o} V$ : coefficient of variation (-)

$M$ : mixing rate (\%)

$n$ : number of pixels (-)

$n^{\prime}$ : index defined by Equation (1) (-)

$R$ : Red brightness (-)

$R_{\max }^{\prime}:$ extracted-maximum brightness (-)

$\gamma$ : shear rate $\left(\mathrm{s}^{-1}\right)$

$\eta$ : apparent viscosity $(\mathrm{Pa} \cdot \mathrm{s})$

$\sigma$ : standard deviation of brightness (-) 